\title{
64-Slice spiral computed tomography and three-dimensional reconstruction in the diagnosis of cystic pancreatic tumors
}

\author{
ZHAOXIA WEN, FENGQING YAO and YUXING WANG \\ Department of Radiology, Linyi People's Hospital, Linyi, Shandong 276003, P.R. China
}

Received November 6, 2014; Accepted December 15, 2015

DOI: $10.3892 /$ etm.2016.3031

\begin{abstract}
The present study aimed to describe the characteristics of cystic pancreatic tumors using computed tomography (CT) and to evaluate the diagnostic accuracy (DA) of post-imaging three-dimensional (3D) reconstruction. Clinical and imaging data, including multi-slice spiral CT scans, enhanced scans and multi-faceted reconstruction, from 30 patients with pathologically confirmed cystic pancreatic tumors diagnosed at the Linyi People's Hospital between August 2008 and June 2014 were retrospectively analyzed. Following the injection of Ultravist ${ }^{\circledR} 300$ contrast agent, arterial, portal venous and parenchymal phase scans were obtained at 28, 60 and $150 \mathrm{sec}$, respectively, and 3D reconstructions of the $\mathrm{CT}$ images were generated. The average age of the patients was 38.4 years (range, 16-77 years), and the cohort included 5 males and 25 females (ratio, 1:5). The patients included 8 cases of mucinous cystadenoma (DA), $80 \%]$; 9 cases of cystadenocarcinoma (DA, 84\%); 6 cases of serous cystadenoma (DA, 100\%); 3 cases of solid pseudopapillary tumor (DA, 100\%); and 4 cases of intraductal papillary mucinous neoplasm (DA, 100\%). 3D reconstructions of CT images were generated and, in the 4 cases of intraductal papillary mucinous neoplasm, the tumor was connected to the main pancreatic duct and multiple mural nodules were detected in one of these cases. The DA of the 3D-reconstructed images of cystic pancreatic tumors was $89.3 \%$. The 64 -slice spiral CT and 3D-reconstructed CT images facilitated the visualization of cystic pancreatic tumor characteristics, in particular the connections between the tumor and the main pancreatic duct. In conclusion, the 3D reconstruction of multi-slice CT data may provide an important source of information for the surgical team, in combination with the available clinical data.
\end{abstract}

Correspondence to: Dr Yuxing Wang, Department of Radiology, Linyi People's Hospital, 27 Jiefang Road, Linyi, Shandong 276003, P.R. China

E-mail: yuxingwangen@126.com

Key words: cystic tumors of the pancreas, cystic and solid masses, multi-slice spiral computed tomography

\section{Introduction}

Cystic tumors of the pancreas account for 5-15\% of pancreatic cystic lesions (1) and $5 \%$ of pancreatic tumors $(2,3)$. Due to the development of imaging technology and an increase in health awareness, cystic pancreatic tumor screening rates have improved (4). The treatment and prognosis of cystic pancreatic tumors with distinct origins is variable, and due to the lack of specific clinical symptoms and laboratory tests available for cystic pancreatic tumors, imaging is particularly important.

The spatial and density resolution of multi-slice computed tomography (CT) is high (5). This technique has been used in fractures $(6,7)$, lymph node metastasis of gastrointestinal cancer (8), pre-operative evaluation of pancreatic cancer (9), and pre-operative staging of esophageal cancer (10). However, the literature on cystic pancreatic tumors is limited. Following image acquisition, multi-slice spiral $\mathrm{CT}$ data can be processed to generate multiplanar reformation and three-dimensional (3D)-reconstructed images. With minimum intensity projection (MinIP) and cured planar reformation (CPR), a bent duct can be transformed into a straight duct, facilitating improved visualization of the connection between the tumor and the pancreatic duct. Furthermore, maximum intensity projection (MIP) can help to visualize the relationship between tumor and adjacent vessels. Overall, 3D reconstruction techniques may provide an important source of information for the surgical team as they can aid the visualization of: Tumor location, size and shape; the pancreatic wall; cavity wall nodules; calcifications; the main pancreatic duct; the connections between the tumor and the pancreatic duct; and the associations between the tumor and the adjacent organs and blood vessels (11). On the basis of these advantages, multi-slice CT may become the standard diagnostic method for cystic pancreatic tumors (12).

In the present study, imaging and clinical data gathered from 30 patients with cystic pancreatic tumors were retrospectively analyzed. The value of $3 \mathrm{D}$ reconstructions of multi-slice spiral CT images for the diagnosis of cystic pancreatic tumors is also discussed.

\section{Materials and methods}

Patients and data collection. CT and clinical data were collected from 30 patients with pathologically confirmed cystic pancreatic tumors in Linyi People's Hospital (Linyi, China) from August 2008 to July 2014. Clinical data are 
Table I. Clinical data of 30 cases of pancreatic cystic lesions in patients with solid tumors.

\begin{tabular}{|c|c|c|c|c|c|c|}
\hline Tumor category & $\begin{array}{l}\text { No. of } \\
\text { cases }\end{array}$ & Gender & $\begin{array}{l}\text { Age } \\
\text { (years) }\end{array}$ & $\begin{array}{l}\text { Average tumor } \\
\text { diameter }(\mathrm{cm})\end{array}$ & Tumor site & Symptoms and signs \\
\hline Serous cystadenoma & 6 & Female & $45-71$ & $2.5-4.4$ & $\begin{array}{l}\text { Pancreatic head }(n=4) \text {, } \\
\text { body and tail }(n=2)\end{array}$ & $\begin{array}{l}\text { Abdominal pain }(n=4) \\
\text { and asymptomatic }(n=2)\end{array}$ \\
\hline Mucinous cystadenoma & 8 & Female & $38-63$ & $5.5-10.5$ & $\begin{array}{l}\text { Pancreatic head }(n=2) \text {, } \\
\text { body and tail }(n=6)\end{array}$ & $\begin{array}{l}\text { Abdominal mass }(n=6) \\
\text { and jaundice }(n=2)\end{array}$ \\
\hline $\begin{array}{l}\text { Mucinous } \\
\text { cystadenocarcinoma }\end{array}$ & 9 & $\begin{array}{l}1 \text { male, } \\
8 \text { female }\end{array}$ & $41-69$ & $3.1-8.7$ & $\begin{array}{l}\text { Pancreatic head }(n=2) \text {, } \\
\text { body and tail }(n=7)\end{array}$ & $\begin{array}{l}\text { Abdominal mass }(n=3) \\
\text { and nausea }(n=6)\end{array}$ \\
\hline Solid pseudopapilloma & 3 & Female & $16-29$ & $5.6-10.8$ & $\begin{array}{l}\text { Pancreatic head }(n=2) \text {, } \\
\text { body and tail }(n=1)\end{array}$ & $\begin{array}{l}\text { Abdominal pain }(\mathrm{n}=1) \\
\text { and asymptomatic }(\mathrm{n}=2)\end{array}$ \\
\hline $\begin{array}{l}\text { Intraductal papillary } \\
\text { mucinous tumors }\end{array}$ & 4 & Male & $60-77$ & $1.5-5.8$ & Pancreatic head $(n=4)$ & $\begin{array}{l}\text { Abdominal pain }(n=2) \\
\text { and jaundice }(n=2)\end{array}$ \\
\hline
\end{tabular}

presented in Table I. The present study was approved by the Ethics Committee of Linyi People's Hospital, in accordance with the Declaration of Helsinki. Written informed consent was obtained from all participants.

CT scan preparation. Following an 8-h fasting period, patients drank $600-800 \mathrm{ml}$ warm water $30 \mathrm{~min}$ before checking in, plus 400-600 $\mathrm{ml}$ warm water immediately prior to lying on the CT bed, in order to fill up the stomach and the ileum.

CT data were acquired at 5-mm intervals from the top of the diaphragm to the bottom of the uncinate process using a LightSpeed ${ }^{\circledR}$ Pro 64-slice CT scanner (GE Healthcare Life Sciences, Waukesha, WI, USA). A high-pressure syringe was used to inject a bolus of 80-100 ml Ultravist ${ }^{\circledR} 300$ contrast agent (Bayer AG, Leverkusen, Germany) into the cubital vein (injection speed, 2.5-3 ml/sec). Following injection of the contrast agent, arterial, portal venous and parenchymal phase scans were obtained at 28,60 and $150 \mathrm{sec}$, respectively.3D reconstruction was performed by constructing two- and three-dimensional images using the original thin-layer $(0.625 \mathrm{~mm})$ volume data, which was subsequently used to assess pathological changes in the pancreatic duct. The average tumor diameter was calculated as the average of the maximum diameter of the tumor cross-section. The diagnostic accuracy (DA) of the 3D-reconstructed images of cystic pancreatic tumors was calculated as: Patients pathologically confirmed following operation/number of patients diagnosed using CT prior to operation x $100 \%$.

\section{Results}

Imaging of mucinous cystadenoma. Among the 30 patients, there were 8 cases of mucinous cystadenoma (DA, 80\%; Fig. 1). Four of these cases demonstrated multiple cystic lesions, of which 2 cases demonstrated uniform wall and cyst spacing and the other 2 cases exhibited uneven wall and cyst spacing with mural nodules. The remaining 4 cases demonstrated a single cystic lesion with uniform thickness. All cysts were large (diameter, $>2 \mathrm{~cm}$ ) and of uneven density with CT values slightly higher than that of water. In 1 case, the cysts contained flocculent material. Following contrast-enhancement, the CT values for the density of the walls increased from 20 to $25 \mathrm{HU}$. No enhancement was detected in the cavity. No tumor inva- sion into the adjacent tissues or connection to the expanded main pancreatic duct were detected. Two cases with mural nodules were misdiagnosed as cystic adenocarcinoma prior to surgery.

Imaging ofmucinous cystadenocarcinoma. The 30 patients in the present study included 9 cases of mucinous cystadenocarcinoma (DA, 84\%; Fig. 2). Five cases demonstrated multiple sac-like cystic lesions with varying sizes, whereas a single cystic lesion was detected in the remaining 4 cases. In all 9 cases, the walls were thick and uneven, including 2 cases with mural nodules; 2 cases with blotchy walls, crude linear calcification, enhanced wall and wall nodules, significantly enhanced solid tissues (contrast-enhanced CT value was increased to 40-45 HU), and unclear wall boundaries; and 2 cases with dilated main pancreatic ducts, one of which exhibited an enlarged para-aortic lymph node. Two cases were misdiagnosed as mucinous cystadenoma as a result of unclear enhancement of the wall.

Imaging of serous cystadenoma. The study group included 6 cases of serous cystadenoma (DA, 100\%; Fig. 3). Three cases exhibited multiple cystic lesions; 2 cases had honeycomb-like cysts; and 1 case had a single cystic lesion. Wall thickness and wall and cyst spacing were uniform in all 6 cases and no wall nodules were detected. Eggshell-like punctate calcifications were detected in 2 cases. The density of the cysts was consistent, with CT values similar to that of water. Following enhanced CT, the density of the walls and the capsule interval were mildly enhanced and the CT value increased to 12-16 HU, however there was no enhancement detected in the cavity. Furthermore, the main pancreatic duct was not dilated and there was no invasion into the adjacent tissues. 3D-reconstructed images demonstrated that the tumors were not connected to the main pancreatic duct (DA, 86.7\%).

Imaging of solid pseudopapilloma. Three cases of solid pseudopapilloma (DA, 100\%; Fig. 4) were investigated in the present study. Two cases demonstrated cystic masses and patchy calcification in the solid tissues, and 1 case exhibited a single cystic lesion, a thicker wall and flocculent material in the cysts. Following enhanced CT, 1 case demonstrated enhancement of the CT value in the wall and the solid tissues of the portal 
A

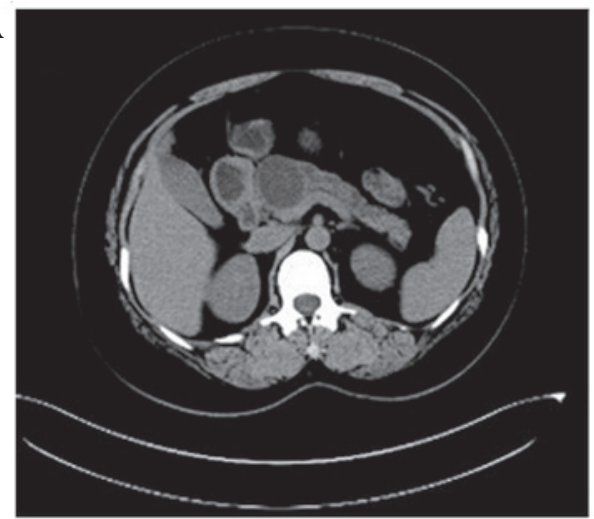

C

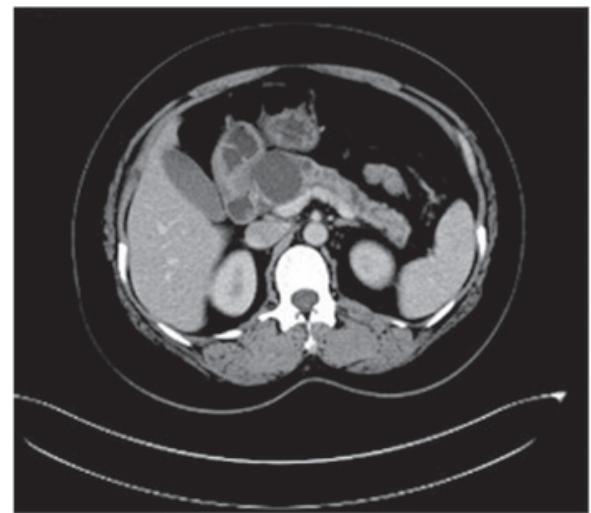

B

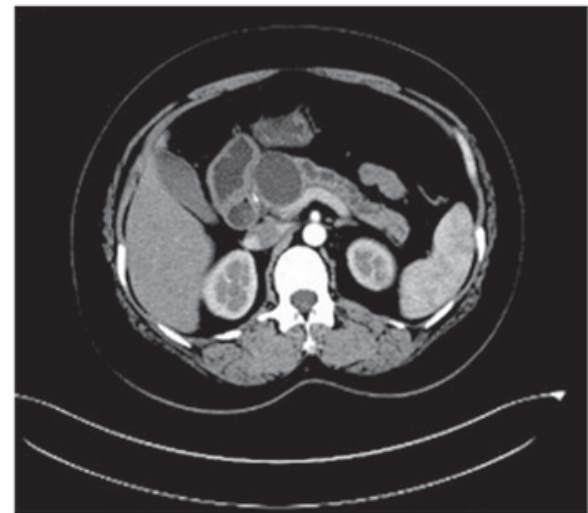

D

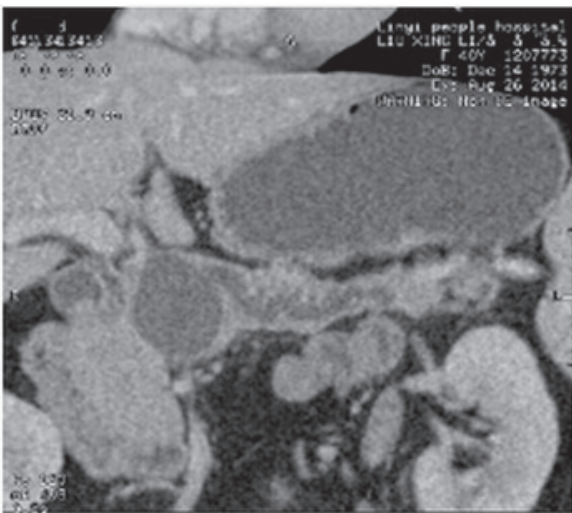

Figure 1. Computed tomography (CT) images of mucinous cystadenoma. (A) Plain CT scan demonstrated a single cystic lesion with clear borders and uniform wall thickness in the uncinate process of the pancreas. (B and C) Enhanced CT examination showed mild enhancement of the wall, with no enhancement of the cyst detected. (D) Reconstruction of minimum intensity projection and cured planar reformation demonstrated that the main pancreatic duct had expanded but did not communicate with the cystic lesion.

$\mathbf{A}$

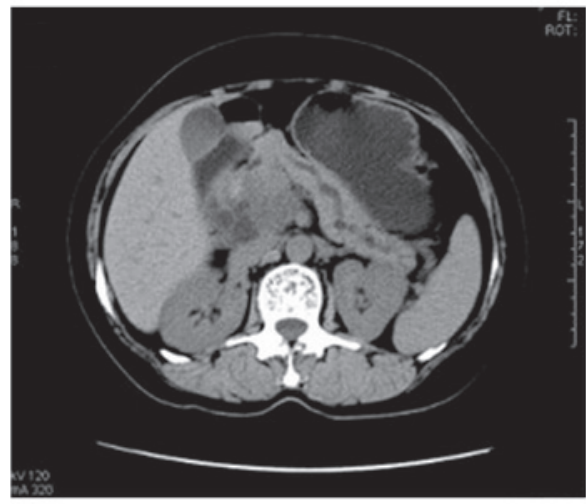

C

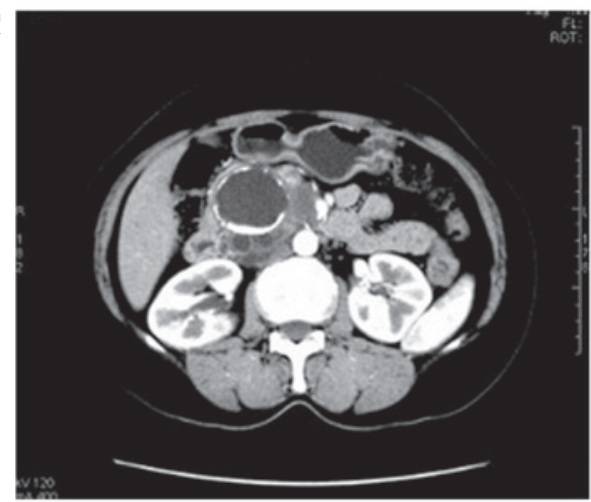

B

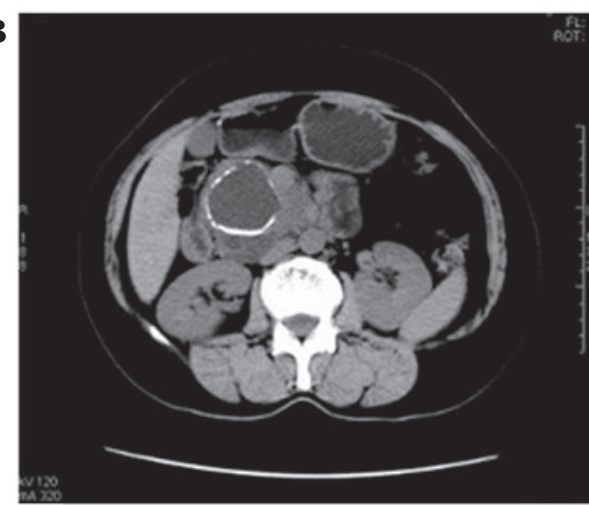

D

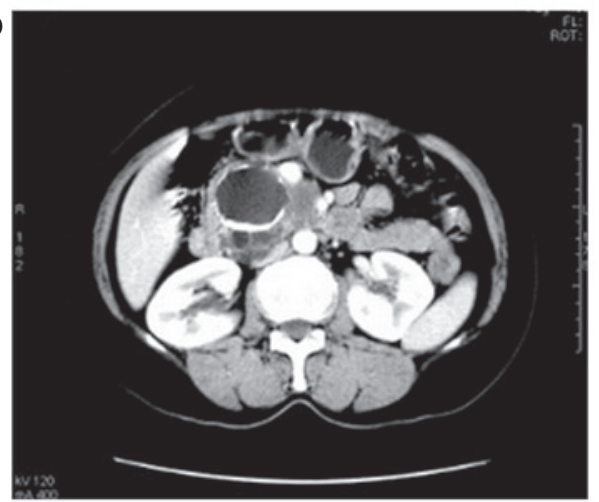

Figure 2. Computed tomography (CT) images of mucinous cystadenocarcinoma. (A and B) Plain CT scans demonstrated multiple cystic lesions in the uncinate process of the pancreas. The sizes of the capsules varied and the wall of the largest cyst demonstrated eggshell calcification; the walls and intervals were thick and varied in thickness. (C and D) Enhanced CT examination demonstrated evident enhancement, whereas no enhancement was detected in the cysts. The main pancreatic had expanded, but do not communicate with the cystic lesions. Lesions surrounded the superior mesenteric vein and had unclear boundaries with the abdominal aorta and inferior vena cava. 
A

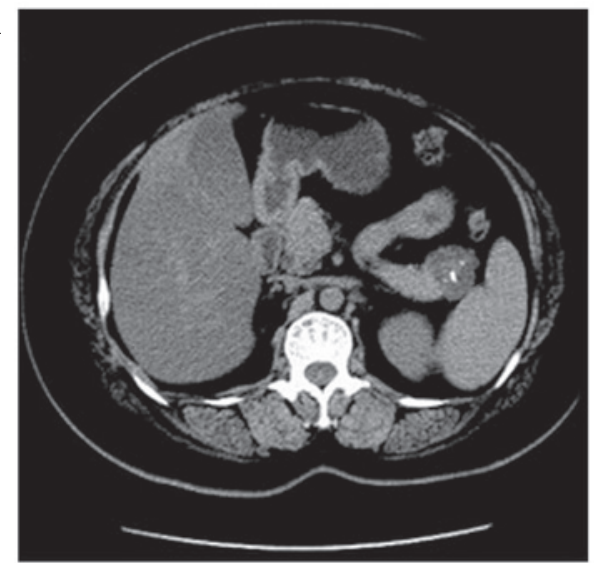

C

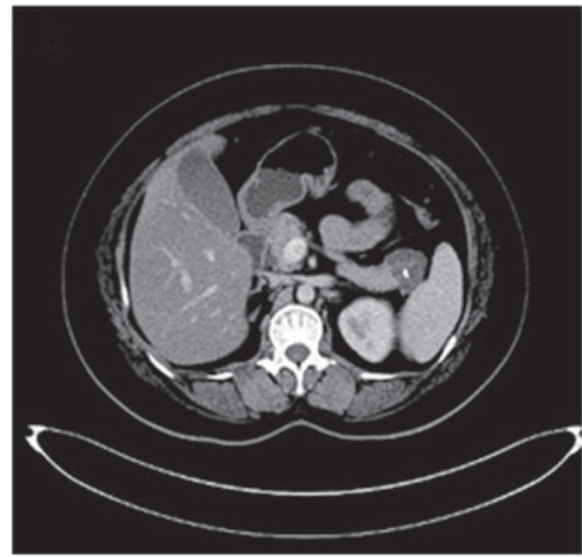

B

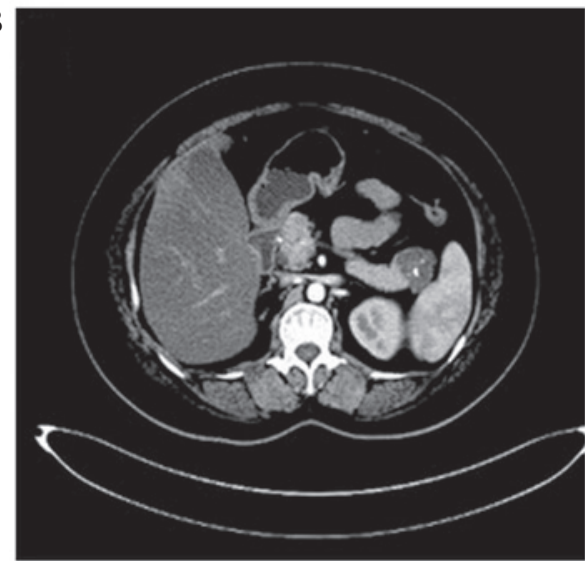

D

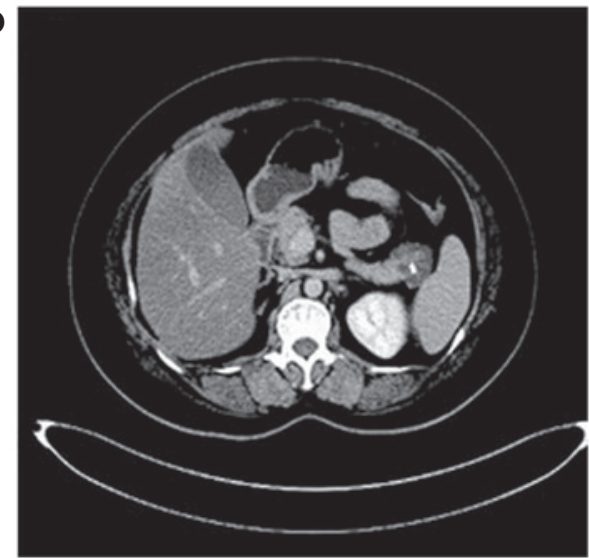

Figure 3. Computed tomography (CT) images of serous cystadenoma. (A) Plain CT scanning demonstrated multiple cystic lesions in the tail of the pancreas. The sizes of capsules varied, the wall and intervals were thin and of uniform thickness, and punctate calcification was detected in the intervals. (B-D) Enhanced CT examination demonstrated mild enhancement; however, no expansion of the main pancreatic duct was detected and the main pancreatic duct did not connect with the cystic lesions.

A

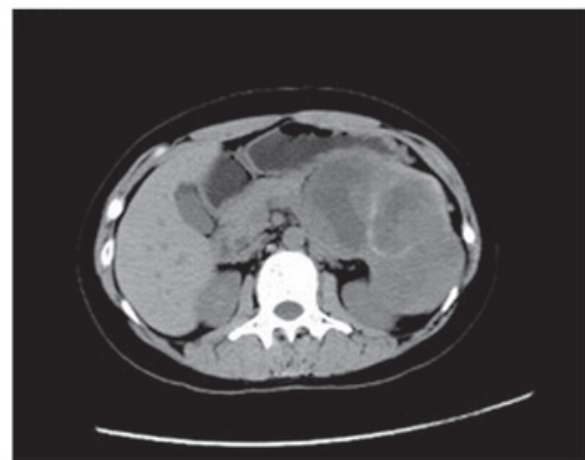

C

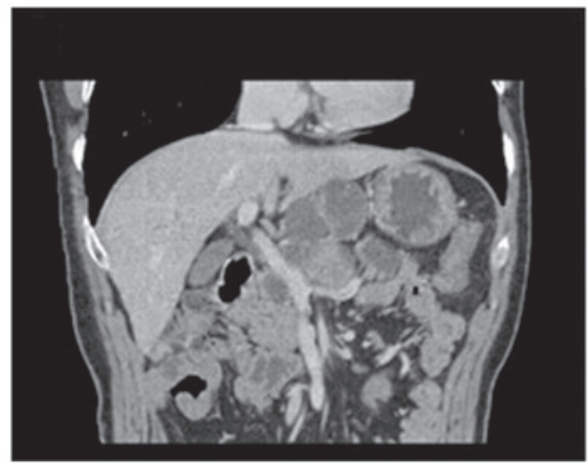

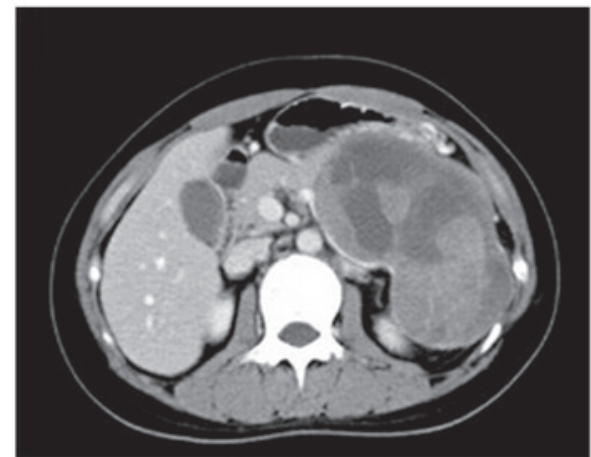

D

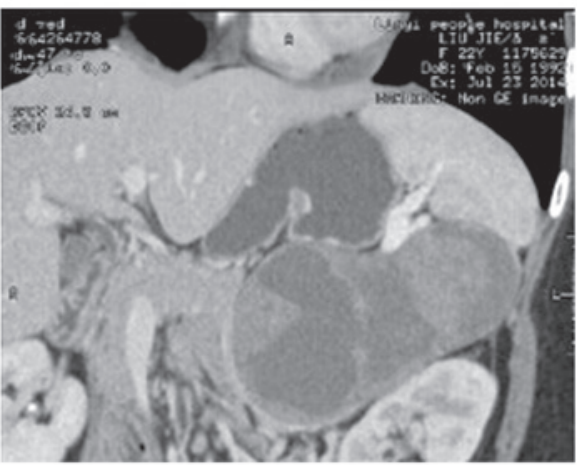

Figure 4. Computed tomography (CT) images of solid pseudopapilloma. (A) Plain CT scanning demonstrated large cystic pancreatic tumors in the tail of the glands and light flaky calcification was detected in the solid area. (B) CT enhancement moderately enhanced the portal venous phase, but not the cysts. (C) Reconstruction of the minimum intensity projection (MIP) showed that the fat gap between the tumor and the superior mesenteric and portal veins had disappeared. (D) Reconstruction of the cured planar reformation indicated that the main pancreatic duct had not expanded and did not connect with the cystic lesions. 

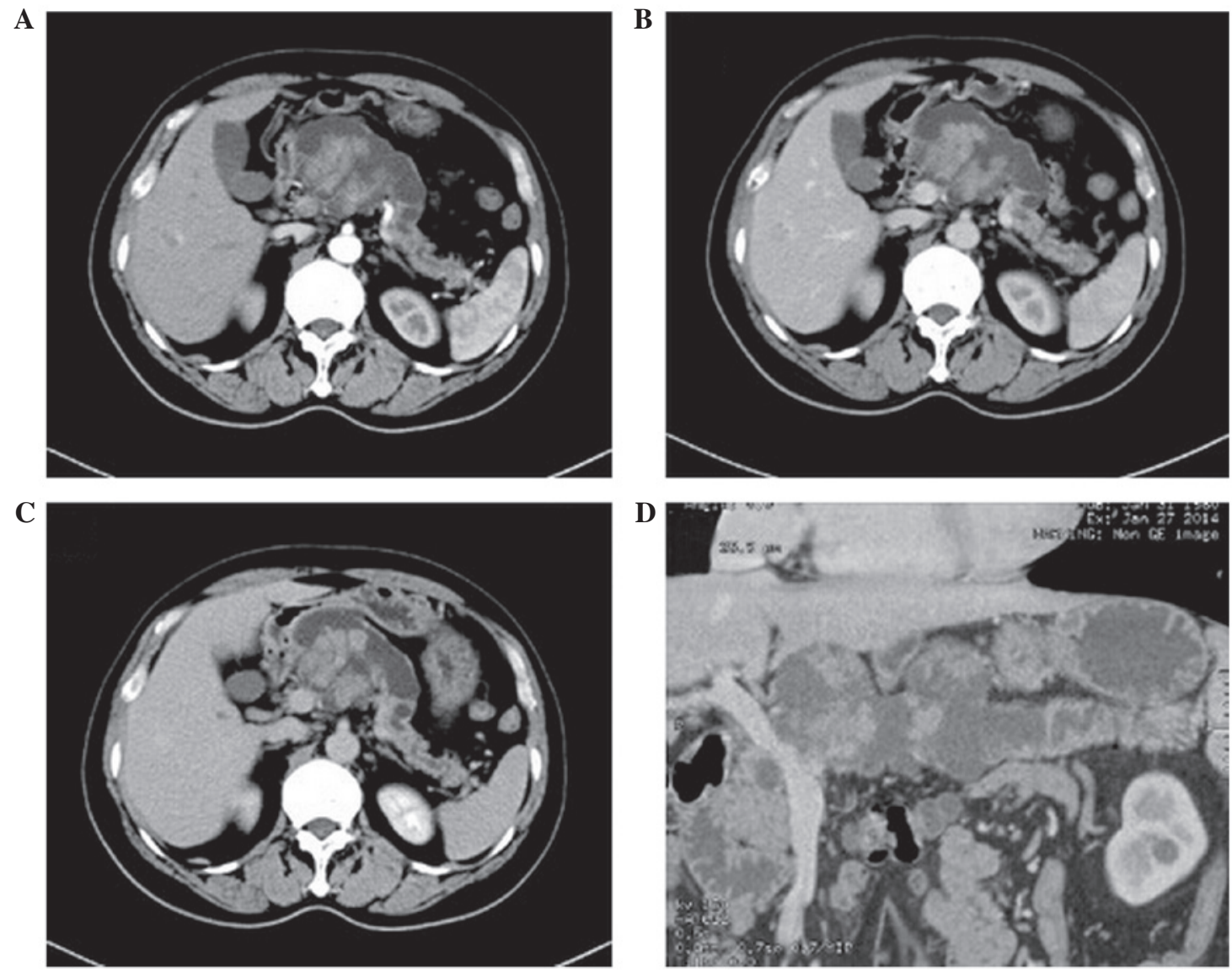

Figure 5. Computed tomography (CT) images of intraductal papillary mucinous tumors. CT enhancement of the (A) arterial, (B) portal and (C) parenchymal phases demonstrated diffuse dilatation of the main pancreatic duct with a cystic lesion in the pancreatic head, and multiple papillary nodules in the cavity. Enhanced examination demonstrated moderate enhancement; the density of the cysts was less uniform with no enhancement. (D) Reconstruction of the minimum intensity projection and cured planar reformation demonstrated that the main pancreatic duct and papillary nodules were dilated.

venous phase were highly enhanced in 2 cases. The $\mathrm{CT}$ values increased to 15 and $23 \mathrm{HU}$, respectively.

Imaging of intraductal papillary mucinous tumors. The remaining 4 patients had intraductal papillary mucinous tumors (DA, 100\%; Figs. 5 and 6). In 2 cases, a single cystic lesion was detected, the walls were not thick, multiple low-density tissue nodules were present on the wall and the cysts were connected to the expanded main pancreatic duct. The other 2 cases demonstrated grape-like cysts, which were connected to the expanded main pancreatic duct. Contrast-enhanced CT images of the wall and the nodules demonstrated persistent enhancement.

\section{Discussion}

Although the incidence of cystic pancreatic tumors is low, these tumors are perceived as increasingly more important among clinicians. According to the World Health Organization (WHO), cystic pancreatic tumors are divided into three categories based on their biological behavior: Benign tumors, malignant tumors and precancerous lesions (13). Cystic tumors of the pancreas are subsequently divided into serous cystadenoma, mucinous cystic neoplasms, solid pseudopapilloma and intraductal papillary mucinous tumors (13-16). The correct preoperative diagnosis of cystic pancreatic tumors determines their treatment and surgical management $(17,18)$.
Mucinous cystadenoma, also known as large cystadenoma, is the most common type of cystic pancreatic tumor, accounting for $\sim 40 \%$ of cystic pancreatic tumors and $2-3 \%$ of primary pancreatic tumors (19). Mucinous cystadenoma occurs most frequently in middle-aged women, with $75 \%$ of these tumors occurring in the 40-60 age range, and anatomically, it occurs both in the body and tail of the pancreas (19). As a precancerous lesion, mucinous cystadenoma often requires surgery. In the present study, 17 cases of mucinous cystic neoplasms were investigated, of which 8 cases were benign and 9 cases were malignant. The CT scans of the majority of the cases demonstrated large, round cystic lesions with a single vesicle diameter $>2 \mathrm{~cm}$. In certain cases, mural nodules were visible in the cavity; eggshell calcification in the wall was visible in $10-25 \%$ cases, which is a characteristic change of the disease (20). When subjected to enhanced examination, the walls, mural nodules and intracapsular intervals were moderately enhanced. In general, the cysts were unevenly spaced, the capsules were thick with mural nodules, and the CT signals of the tissues were significantly enhanced.

Pancreatic duct obstruction may be associated with localized mucinous cystadenocarcinoma (4); however, the results of the present study suggested that the expansion of the pancreatic duct and the lesion do not appear to be associated. In the present study, 2 cases of mucinous cystadenoma were misdiagnosed as cystic adenocarcinoma due to the presence 
$\mathbf{A}$

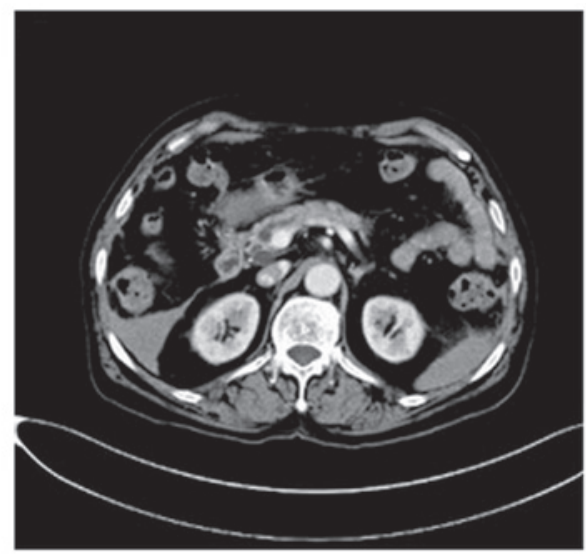

C

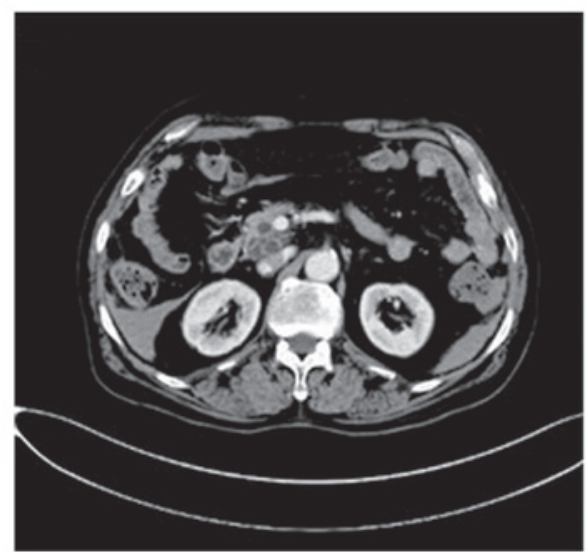

B
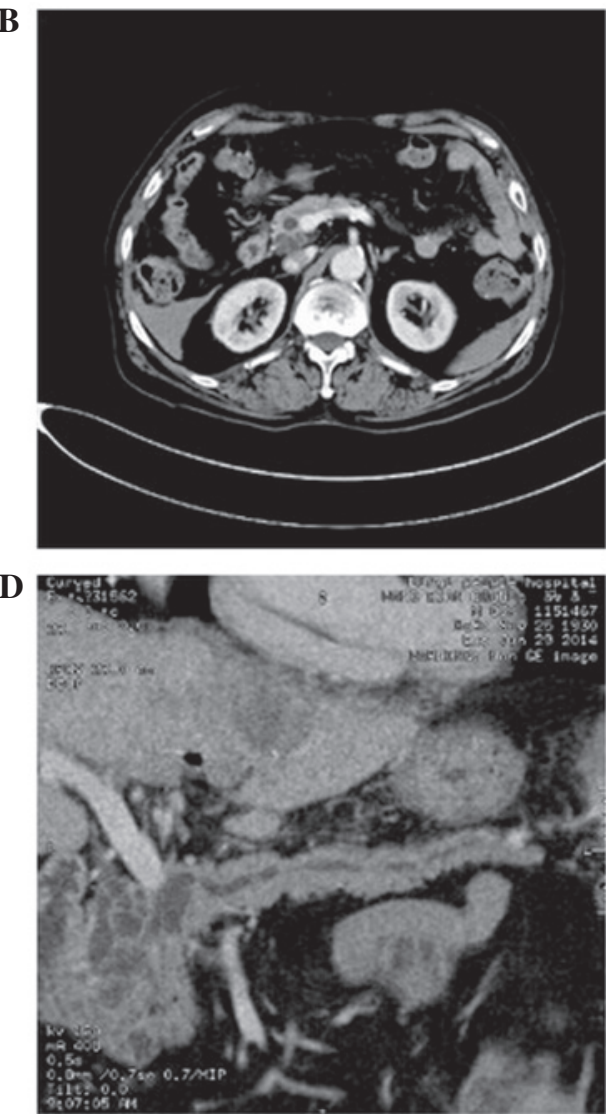

Figure 6. Computed tomography (CT) images of intraductal papillary mucinous tumors. (A-C) CT enhancement detected multiple cystic lesions in the uncinate process of the pancreas, the interval was visible and the cysts were small. Enhanced examination demonstrated mild enhancement and revealed that the main pancreatic duct was expanded. (D) Reconstruction of the minimum intensity projection and cured planar reformation showed grape-like lesions that communicated with the expansion of the main pancreatic duct.

of mural nodules and moderate enhancement on the CT. Furthermore, 2 cases of bladder cancer were misdiagnosed as mucinous cystadenoma due to slight enhancement of the wall. The CT scans of mucinous cystadenoma and cystadenocarcinoma were similar, indicating that there may be potential difficulties in differential diagnosis between these tumor types.

Serous cystadenoma, also known as microcapsules or small cystic adenoma, is the second most common type of cystic pancreatic tumor, accounting for $\sim 30 \%$ of cystic pancreatic tumors and 1-2\% of primary pancreatic tumors (4). Serous cystadenoma tumors originate in the central acinar and tubular cells and are typically located in the pancreatic head (14). They are most frequent in women aged $\geq 60$ years (male:female ratio, 1:4) (14). As serous cystadenomas are benign tumors which rarely undergo malignant transformation, surgery is only indicated for large tumors (14). Among the 6 cases of serous cystadenoma evaluated in the present study, 4 cases occurred in women aged 60-70 years and were located in the pancreatic head, whereas 2 cases occurred in the pancreatic tail. The majority of cases exhibited multiple cystic lesions with a mean diameter of $5 \mathrm{~cm}$ (range, 1.4-27 cm) and pale lobulated edges, and multiple $(>6)$ vesicles (diameter, $0.2-2 \mathrm{~cm}$ ). Furthermore, $\sim 33.3 \%$ of cases exhibited a honeycomb-like appearance; $20 \%$ of cases exhibited a single cystic lesion or cystic lesions $>2 \mathrm{~cm}$ in diameter; and $30 \%$ of cases exhibited a central fibrous scar, with or without astral calcification, with slight enhancement of the wall and capsule and no association with dilatation of the pancreatic duct.

Comparison of the aforementioned observations for serous and mucinous cystadenoma indicates that mucinous cystadenoma typically occurs in younger patients (40-60 years old) and is located in the body and tail of the pancreas, with large vesicles (diameter, $>2 \mathrm{~cm}$ ), and thick-walled and unevenly spaced cysts with a density higher than that of water, whereas serous cystadenoma typically occurs in patients $\geq 60$ years old and is located in the head of pancreas, with a small capsule (diameter, $<2 \mathrm{~cm}$ ), thin walls, and cysts with a density similar to that of water. Astral calcification is a typical characteristic of serous cystadenoma.

As compared with serous and mucinous cystadenoma, solid pseudopapilloma has a lower morbidity and accounts for $5 \%$ of cystic pancreatic tumors and $0.9-2.7 \%$ of primary pancreatic tumors (21). Notably, $91 \%$ of these tumors occur during adolescence or in young women (average age, 28 years; male:female ratio, 1:9), and are often located in the head and tail of the pancreas (21). As a benign or low-grade malignant tumor, solid pseudopapilloma requires surgical treatment (21). CT examination showed large cystic, solid lesions (diameter, 2.5-25 cm; general diameter, $>5 \mathrm{~cm}$ ), which were often associated with bleeding within the tumor and enlarged reactive lymph nodes; $66.7 \%$ of cases demonstrated pleomorphic calcifications. On enhanced CT examination, the enhancement of the portal venous phase was elevated, as compared with the arterial phase. 
Intraductal papillary mucinous tumors account for $20 \%$ of cystic pancreatic tumors and $0.5-9.8 \%$ of primary pancreatic tumors (22). These tumors are more frequent in elderly men with $87 \%$ occurring in the 60-70 year age range (4). They originate in the ductal epithelium and are divided into three types, namely main duct, branch duct and mixed. Branch duct intraductal papillary mucinous tumors most commonly occur in the uncinate process of the pancreas, and exhibit similarities with the main pancreatic duct type in terms of grape-like cysts, cystic masses and a dilated branch duct. CT scans of the main pancreatic duct type typically demonstrate diffuse main pancreatic duct or localized expansion, with surrounding pancreatic atrophy and papillary nodules within the pancreatic duct (4). Furthermore, multiple mural nodules, which are the solid components of tumors, expand into the main pancreatic duct and thus likely contribute to tumor malignancy (23). Mixed lesions are associated with the main pancreatic duct and branches. In order to confirm the relative locations of cystic pancreatic tumors in the present study, MinIP and CPR techniques were applied, which may aid the differential diagnosis of these tumors. In particular, it is important that branch-type intraductal papillary mucinous and serous tumors are identified $(24,25)$, as branch-type intraductal papillary mucinous tumors occur in older men and are characterized by a dilated pancreatic duct that communicates with the tumor. By contrast, serous cystadenoma often occurs in older women, without dilatation or communication with the pancreatic duct. Therefore, the differential diagnosis of cystic pancreatic tumors should consider gender, age, anatomical location, cavity size, number of cysts, wall thickness, wall nodules and duct morphology data.

The present study demonstrated that, as a non-invasive, convenient and widely applied imaging method, multi-slice CT can provide detailed information on pancreatic cystic lesions. Although cystic tumors demonstrate similar CT changes, this imaging technique facilitates a more accurate diagnosis of cystic pancreatic tumors and provides important information for their surgical management. As the sample size of the present study was small, future studies should be conducted with larger patient samples.

\section{References}

1. Wilentz RE, Albores-Saavedra J and Hruban RH: Mucinous cystic neoplasms of the pancreas. Semin Diagn Pathol 17: 31-42, 2000.

2. Cubilla AL and Fitzgerald PJ: Classification of pancreatic cancer (nonendocrine). Mayo Clin Proc 54: 449-458, 1979.

3. Sener SF, Fremgen A, Imperato JP, Sylvester J and Chmiel JS: Pancreatic cancer in Illinois. A report by 88 hospitals on 2,401 patients diagnosed 1978-84. Am Surg 57: 490-495, 1991.

4. Limaiem F, Khalfallah T, Farhat LB, Bouraoui S, Lahmar A and Mzabi S: Pancreatic cystic neoplasms. N Am J Med Sci 6 : 413-417, 2014.

5. Hara T, Kato H, Akiyama M and Murata K: Basic examination of in-plane spatial resolution in multi-slice CT. Nihon Hoshasen Gijutsu Gakkai Zasshi 58: 473-478, 2002 (In Japanese).

6. YG, Tan YL, Mo JC, Zheng RB, Ye DK, Wu D, Luo DL and Peng S: Comparison of diagnostic value between DR and MSCT in fracture and dislocation of foot and ankle. Zhongguo $\mathrm{Gu}$ Shang 26: 553-556, 2013 (In Chinese).
7. Devireddy SK, Kumar RV, Gali R, Kanubaddy SR, Rao DM and Siddhartha M: Three-dimensional assessment of unilateral subcondylar fracture using computed tomography after open reduction. Indian J Plast Surg 47: 203-209, 2014.

8. Dai CL, Yang ZG, Xue LP and Li YM: Application value of multi-slice spiral computed tomography for imaging determination of metastatic lymph nodes of gastric cancer. World $\mathrm{J}$ Gastroenterol 19: 5732-5737, 2013.

9. Rebibo L, Chivot C, Fuks D, Sabbagh C, Yzet T and Regimbeau JM: Three-dimensional computed tomography analysis of the left gastric vein in a pancreatectomy. HPB (Oxford) 14: 414-421, 2012.

10. Cheng ZZ, Yang NJ, Xi XQ, Zhao K, Hu SB, Xu GH, Ren J and Zhou P: Diagnostic and application value of 64-slice spiral CT scanning in preoperative staging of esophageal cancer. Zhonghua Zhong Liu Za Zhi 33: 929-932, 2011 (In Chinese).

11. Yu Y, Guo M and Han X: Comparison of multi-slice computed tomographic angiography and dual-source computed tomographic angiography in resectability evaluation of pancreatic carcinoma. Cell Biochem Biophys 70: 1351-1356, 2014.

12. Yoon SE, Byun JH, Kim KA, Kim HJ, Lee SS, Jang SJ, Jang YJ and Lee MG: Pancreatic ductal adenocarcinoma with intratumoral cystic lesions on MRI: Correlation with histopathological findings. Br J Radiol 83: 318-326, 2010.

13. Hruban R, Klöppel G, Boffetta P, Maitra A, Hiraoka N and Offerhaus GJA: Tumours of the pancreas. In: WHO Classification of Tumours of the Digestive System. Bosman T, Carneiro F, Hruban R and Theise ND (eds). Vol 3. 4th edition. IARC Press, Lyon, pp280-330, 2010.

14. Kosmahl M, Pauser U, Peters K, Sipos B, Lüttges J, Kremer B and Klöppel G: Cystic neoplasms of the pancreas and tumour-like lesions with cystic features: A review of 418 cases and a classification proposal. Virchows Arch 445: 168-178, 2004.

15. Yoon WJ, Lee JK, Lee KH, Ryu JK, Kim YT and Yoon YB: Cystic neoplasms of the exocrine pancreas: An update of a nationwide survey in Korea. Pancreas 37: 254-258, 2008.

16. Basturk O, Coban I and Adsay NV: Pancreatic cysts: Pathologic classification, differential diagnosis and clinical implications. Arch Pathol Lab Med 133: 423-438, 2009.

17. Klöppel G and Heitz PU: Pancreatic endocrine tumors. Pathol Res Pract 183: 155-168, 1988.

18. Krechler T, Ulrych J, Dvořák M, Hoskovec D, Macášek J, Śvestka T and Hořejš J: Cystic tumors of the pancreas - our experience with diagnostics. Vnitr Lek 59: 572-577, 2013 (In Czech).

19. Hruban RH, Pitman MB and Klimstra DS (eds): AFIP Atlas of Tumor Pathology. Tumors of the Pancreas. Fascicle 6. 4th series. 6th edition. Armed Forces Institute of Pathology, Washington, DC, 2007.

20. Klimstra DS: Cystic, mucin-producing neoplasms of the pancreas: The distinguishing features of mucinous cystic neoplasms and intraductal papillary mucinous neoplasms. Semin Diagn Pathol 22: 318-329, 2005.

21. Ren Z, Zhang P, Zhang X and Liu B: Solid pseudopapillary neoplasms of the pancreas: Clinicopathologic features and surgical treatment of cases. Int J Clin Exp Pathol 15: 6889-6897, 2014.

22. Acar M and Tatli S: Cystic tumors of the pancreas: A radiological perspective. Diagn Interv Radiol 17: 143-149, 2011.

23. Khouri J and Saif MW: Intraductal papillary mucinous neoplasms of the pancreas (IPMNs): New insights on clinical outcomes and malignant progression. JOP 15: 310-312, 2014.

24. Yokoyama S, Sasaki Y, Hashimoto K, Takeda M, Toshiyama R, Fukuda S, Naito A, Matsumoto S, Tokuoka M, Ide Y, et al: A case of invasive ductal carcinoma of the pancreas originating from an intraductal papillary mucinous tumor that was initially misdiagnosed as a mucinous cystic tumor. Gan To Kagaku Ryoho 39: 2149-2151, 2012 (In Japanese).

25. Palmucci S, Trombatore C, Foti PV, Mauro LA, Milone P, Milazzotto R, Latino R, Bonanno G, Petrillo G and Di Cataldo A: The utilization of imaging features in the management of intraductal papillary mucinous neoplasms. Gastroenterol Res Pract 2014: 765451, 2014. 